\title{
An Efficient Collision Free Protocol for VANET
}

\author{
Ghassan Samara \\ Internet Technology Department, \\ Faculty of Information Technology, Zarqa University \\ Zarqa, Jordan
}

\begin{abstract}
VANET is considered as one of the most important research field in the wireless and mobile technology, which provides road safety, updated traffic information, and infotainment. The transmission technology used in VANET is the wireless broadcast which means that the vehicles on the road are sharing a single communication channel for broadcasting the messages, usually VANET suffers from the number of nodes consisting and competing to reserve and use the channel, and this leads to high collision scenarios if the situations are not managed wisely.

In this research a new collision free protocol hereinafter will be called (CF-MAC) to manage the channel access in Medium Access Control Sublayer (MAC) for the transmission will be proposed, this protocol will ensure a collision-free management to enhance the channel performance, and increase the channel reliability. The evaluation criteria will depend on the QoS enhancement like channel throughput, message delay, and message loss; the results show that the overall channel performance in terms of collision and packet loss ratio is improved.
\end{abstract}

\section{Keywords}

VANET, Collision free, Message broadcasting,, CF-MAC.

\section{INTRODUCTION}

Vehicular ad hoc networks (VANETs) is an important research field for the Intelligent Transportation Systems (ITS). Based on IEEE 802.11p [1], VANET has it special wireless radios called Dedicated Short Range Communication (DSRC) that support high-speed communication, high mobile nodes and high bandwidth. The US Federal Communication Commission (FCC) has allocated $75 \mathrm{MHz}$ of the spectrum at $5.9 \mathrm{GHz}$ exclusively for vehicular communications. VANET channel is divided into seven $10 \mathrm{MHz}$ channels [2]. The Channel 178 is the Control Channel $(\mathrm{CCH})$ for the transmission of emergency and status messages (Beacon). The other channels are Service Channels (SCHs), where more data transfers and special applications. Channel 172 and Channel 184 are used for safety applications in Vehicle-to-Vehicle (V2V) communication. The bandwidth can reach 54 Mbps. Many single-channel [3], [4] and [5] MAC protocols have been developed for VANET, where all vehicles share a single communication channel in the network. Wireless Access in Vehicular Environments (WAVE) MAC utilises a contentionbased access using Carrier Sense Multiple Access with Collision Avoidance (CSMA/CA) mechanism. As a result of high contentions and collisions, the performance of singlechannel MAC protocols collapses quickly with the increase in density of vehicles.

Vehicular Ad-hoc Network (VANET) is a network containing a huge number of connected mobile vehicles forming a very dynamical network. VANET is a type of Mobile Ad hoc Networks (MANET), and it does not have an infrastructure or centralized access point. VANET aims to support many safety applications, entertainment and traffic optimization. VANET vehicles usually equipped with communication device like Global Positioning System (GPS) receiver, On-Board Unit (OBU), and a set of stationary units along roads, called Road Side Units (RSUs). Based on OBU and RSU, VANET has two essential communications: Vehicle-to-Vehicle (V2V) in which vehicles communicate with each other on the move and Vehicle-to-RSU (V2R) where the moving vehicle communicate with the fixed RSU.

Many vehicles in the same spot, means more contention, delay and maybe high collision.

Many applications have been developed during the last years (e.g. See [6]) based on the notion of vehicles exchanging Cooperative Awareness Messages (CAM) or beacons, to enhance traffic efficiency and safety or to provide infotainment. Especially the efficiency and safety applications that may come with high requirements with respect to maximum allowable delay and success probability.

One of the reasons why standardization is moving towards the adoption of IEEE 802.11p [7] (or its European counterpart ETSI-G5 [8]) for this purpose is that the behaviour of the 802.11 family is well known. However, existing unicast 802.11 models are not directly applicable to the VANET scenarios.

Beacons are transmitted using the CSMA/CA Broadcast method [9]. Hence it is critical to managing MAC layer behaviour correctly. When designing a model for beaconing in VANETs, the model was presented in [10] and covered the entire saturation spectrum. Even though [10] modelled EDCA, the backoff counter decrement behaviour is that of the DCF. When removing the CW and AIFS differentiation, an accurate model of the DCF remains.

The original IEEE 802.11, was designed for wireless local area networking (WLAN), has two drawbacks within its medium access control (MAC) technique carrier sense multiple access (CSMA): as it may cause delay before channel access, the other problem is the channel collision.

The MAC protocol decides who can reserve the shared channel to transmit when there is a competition for it. In a carrier sense systems, such as CSMA, each sender first listens to the channel and if there is no one is sending for a certain time period, the node transmits directly, at the same time there is a possibility that another node is transmitting and this results in a collision on the channel. Moreover, a node may experience very long channel access delays due to the risk of the channel being busy during its listening period. These two situations occur primarily during high utilization periods in the network. CSMA is used by the whole IEEE802.11 family as well as its wired counterpart IEEE 802.3Ethernet. One of the reasons for the success of both WLAN and Ethernet is the straightforward implementation of the standard resulting in reasonably priced equipment. Due to this WLANs and Ethernet are often applied to other domains than they 
originally were designed for. Even though CSMA is not suitable for real-time communication because of the uncontrolled channel access delays, Ethernet became common in the industrial communication scene where many real-time systems are found. However, the problems with MAC can be solved by using more network devices, such as switches and routers, and hence reducing the number of nodes competing for the shared channel, that is, reducing the collision domains. In the wireless domain, however, there is no easy solution since all nodes share the wireless channel.

Furthermore, when the CSMA algorithm is applied in the wireless, a node could easily jam the network, intentionally or unintentionally, and the nodes in this area would defer their transmissions even though there is no real data traffic. A wireless carrier sense system is thus more prone to interference since no access will occur as long as the activity is detected on the channel.

The IEEE 802.11p standard, which is designed for vehicular ad hoc networks (VANET) [11], uses CSMA as its MAC access, despite that it does not support real-time deadlines. The argument is that the CSMA is producing problems with high traffic networks, and traffic shaping can be used to keep the data traffic at an acceptable level. However, traffic shaping is typically used in centrally controlled networks or networks in restricted geographical areas. Which is not suitable for VANET as it is not restricted to a geographical area and can't be used by a central controller due to its highly dynamic characteristics and requirements on low delay. Also, traffic shaping only reduces the average delay, and the main problem with the delay still exists. A solution to the problem with the channel access delays when using CSMA could be to use a self-organized time division multiple access (STDMA), a decentralized, yet predictable, MAC protocol with a finite channel access delay, making it appropriate for real-time adhoc vehicular networks. An STDMA algorithm is already widely used in a system called automatic identification system (AIS), where it focuses on collision avoidance between ships.

The proposed research aims to enhance the channel performance and increase the channel reliability. Furthermore, the collision resulted from this message will be eliminated.

\section{THE PROPOSED CF-MAC PROTOCOL}

\section{Initial Assumptions:}

In the proposed protocol it is assumed that the following points are fulfilled:

1. $\mathrm{V} 2 \mathrm{~V}$ communication: the communication in the network consists of vehicle to vehicle infrastructure, no roadside units will be engaged [12].

2. Each vehicle transmits a beacon (status) message, 10 messages per a second.

3. All the vehicles inside the channel contend to reserve it to make the transmission when any broadcast happens all other vehicles have to wait for this broadcast to end.

4. Each vehicle forms a Neighbour Table (NT) which contains the ID and the MAC address of all neighbouring vehicles; the received beacons freshly collect this information from neighbours.

5. Vehicles on the roads form the platoon phenomenon.
6. Each vehicle is equipped with GPS device that gives an accurate position.

A dynamic and self-configured TDMA will be used to manage the channel access. Usually, vehicles use the Carrier Sense Multiple Access/ Collision Avoidance CSMA/CA to detect the collision and the transmission in the channel, but this technique is not always suitable in VANET [13], [14] and [15]. As collision happens at the receiver, not at the sender.

Vehicles on the road are moving in a platoon shape [16] and [17] and forming a group of adjacent nodes these nodes sharing a common channel properties, nodes, and same neighbor table which includes the nearby nodes which contend with the current node to reserve the channel, see figure 1 for the platoon and table for the Neighbor Table 1 .

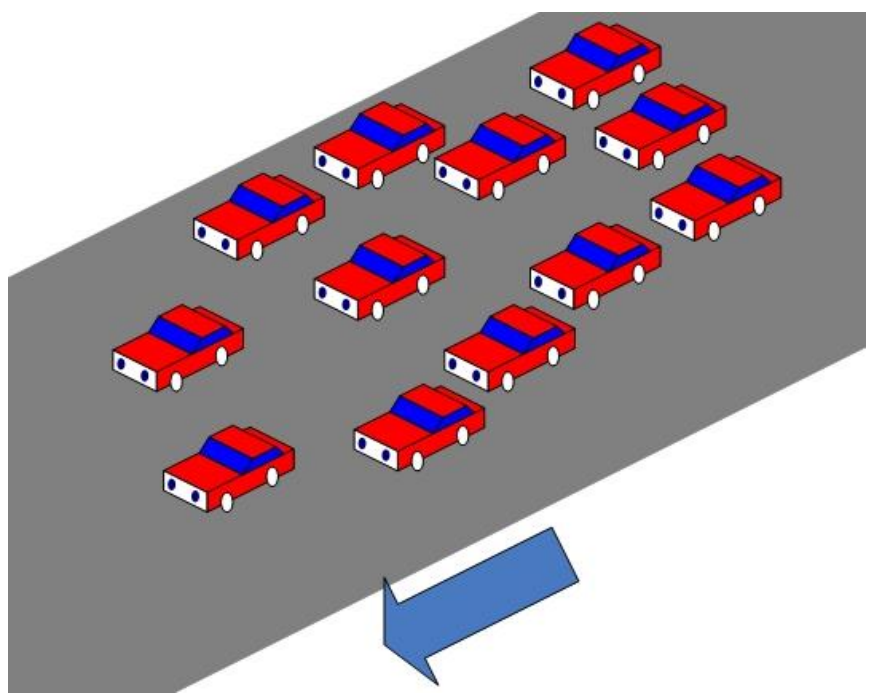

Fig 1: Platoon shape

Table 1: Neighbor Table Structure

\begin{tabular}{|c|c|c|}
\hline MAC Address & Longitude & Latitude \\
\hline $\begin{array}{c}\text { 00-1D-0F-C3-01- } \\
\text { D3 }\end{array}$ & 40.689060 & 74.152456 \\
\hline $\begin{array}{c}\text { 01-1D-3D-C3-78- } \\
\text { F6 }\end{array}$ & 40.344536 & 74.341231 \\
\hline $\begin{array}{c}\text { 02-1D-0F-R6-42- } \\
\text { F2 }\end{array}$ & 41.752370 & 75.000040 \\
\hline $\begin{array}{c}\text { 03-1D-0F-C3-77- } \\
\text { F7 }\end{array}$ & 42.601021 & 74.753527 \\
\hline
\end{tabular}

\section{The protocol steps:}

Any vehicle wants to transmit, it forms an Initial Broadcast Table (IBT), the IBT must be ordered according to the MAC address of each vehicle in ASC order, the MAC address is obtained from NT, the vehicle that initiates the IBT will add its MAC address in the first slot, the rest of the NT contents will be ordered in ascending order, the priority for transmission will be given from the first slots, see table 2 which illustrates the IBT structure.

The fresh IBT will be broadcasted to all neighbouring vehicles when the vehicle is allowed to transmit (i.e. when the vehicle reaches its turn), by receiving this table, all neighbouring vehicle will understand that the channel is reserved for sender vehicle.

When a receiver vehicle receives a new IBT from one of its neighbors; it will scan its first slot to know the initiator, at this moment, if the receiver wishes (wants) to send, it will reply to the initiator by sending a message Want To Send (WTS), the 
initiator upon receiving this message it will add 1 in IBT in the vehicle that wishes to send slot.

Table 2: Initial Broadcast Table

\begin{tabular}{|c|c|}
\hline Vehicle MAC & WTS \\
\hline 01-1D-3D-C3-78-F6 & \\
\hline 02-1D-0F-R6-42-F2 & \\
\hline 00-1D-0F-C3-01-D3 & \\
\hline 03-1D-0F-C3-77-F7 & \\
\hline
\end{tabular}

See figure 2 and 3 that lustrate how the protocol works.

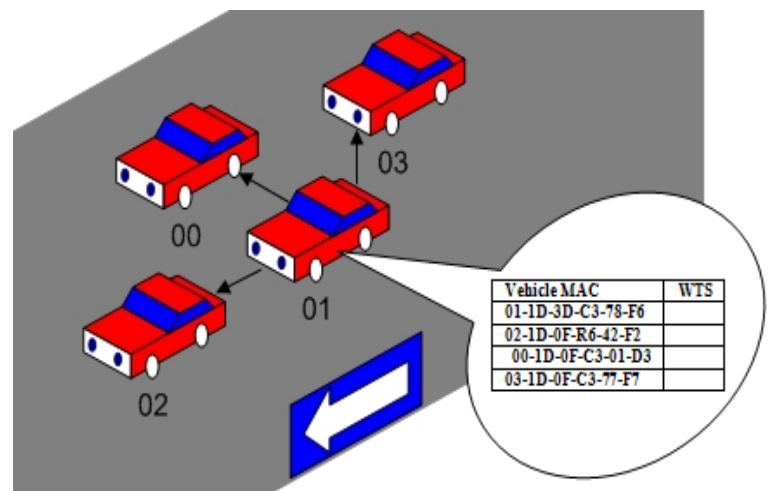

Fig 2: Broadcast Table

Where vehicle 01 initiates the fresh BT and broadcast it to its neighbours, vehicles $(00,02,03)$ will reply if it wants to transmit during this time slot by sending WTS message to vehicle 01 , see figure 3 .

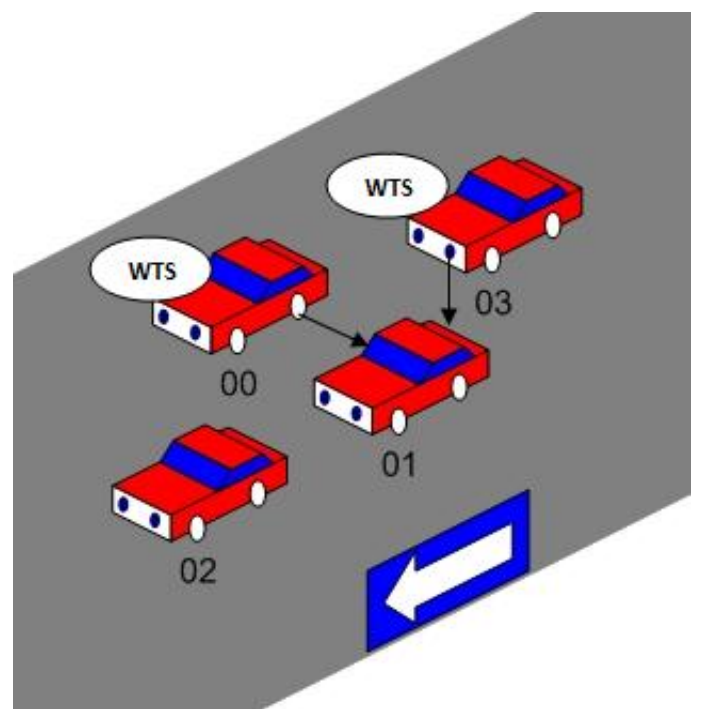

Fig 3: Want to Send

The sending priority at the moment is given to the vehicle that reserved the first slot at $\mathrm{BT}$, so this vehicle will start transmitting its message, the BT will be piggybacked to the transmitted message.

When a receiver receives the message, it will extract it along with the BT; the BT will make it clear who is going to transmit next.

All the vehicles inside the territory know the transmission priority, which is according to the BT, so each vehicle will transmit in its turn by waiting for the vehicle that lies before it in the BT to finish the transmission, see the BT after the ordering in Table 3.

\section{Table 3: Broadcast Table}

\begin{tabular}{|c|c|}
\hline Vehicle MAC & WTS \\
\hline 01-1D-3D-C3-78-F6 & 1 \\
\hline 02-1D-0F-R6-42-F2 & 0 \\
\hline 00-1D-0F-C3-01-D3 & 1 \\
\hline 03-1D-0F-C3-77-F7 & 1 \\
\hline
\end{tabular}

In the previous example, vehicle 01 will start the transmission, and it will add the final BT attached (piggybacked) with the transmitted message, and vehicle 00 will wait for the end of the transmission, in turn, vehicle 03 is waiting for vehicle 00 transmission, after the end of vehicle 03 transmission, the first slot that has 0 in WTS is allowed to transmit its $\mathrm{BT}$, and therefore, vehicle 02 will start transmitting its BT which includes a fresh information about the vehicles neighboring vehicle.

In case that there is no vehicle has 0 in its WTS, any vehicle inside the BT wants to send it must contend with the other BT vehicles that want to send, in the contention the vehicle will wait for a random amount of time (SIFS, DIFS) to avoid any congestion.

For the new vehicles who join the platoon, it should receive the messages and interact with the neighbors once it is requested to do so, it cannot start transmitting as soon it joins the group, so it will receive the transmission from neighbors, once it receives the BT and want to transmit, it will add 1 in its WTS slot and waits for its turn to start transmitting.

\section{SIMULATION RESULTS}

In this works, Matlab R2017a was used to test the correctness of the proposed CF-MAC protocol, the protocol tested and compared with the Distributed TDMA MAC protocol called DTMAC proposed in [18], where the environment and all simulation parameters used in DTMAC were adopted in the proposed protocol CF-MAC.

\section{Simulation Parameters:}

Highway scenario with two lanes of size $2000 \mathrm{~m} \times 20 \mathrm{~m}$, in both directions, the speed of the moving vehicle is constant, the simulation parameters are summarized in table 4 .

Table 4: Simulation parameters.

\begin{tabular}{|c|c|}
\hline Street length & $2 \mathrm{~km}$ \\
\hline Lanes & 2 lanes \\
\hline Speed & $120 \mathrm{~km} / \mathrm{h}$ \\
\hline Transmission range & $300 \mathrm{~m}$ \\
\hline Number of vehicles & 100 \\
\hline Network interface & Phy/WirelessPhyExt \\
\hline MAC interface & Mac/802 11Ext \\
\hline Interface queue & Queue/DSRC \\
\hline Propagation model & Propagation/Nakagami \\
\hline $\begin{array}{c}\text { Number of TDMA } \\
\text { slots/frames }\end{array}$ & 10 \\
\hline Time slot & $2.5 \mathrm{~ms}$ \\
\hline Antenna type & Antenna/omniantenna \\
\hline Channel type & Channel/wireless channel \\
\hline $\begin{array}{c}\text { Minimum beaconing } \\
\text { interval }\end{array}$ & $100 \mathrm{~ms}$ \\
\hline $\begin{array}{c}\text { Maximum beaconing } \\
\text { interval }\end{array}$ & $500 \mathrm{~ms}$ \\
\hline
\end{tabular}

Area occupancy (AO) parameter was used in [19], which is equal to $\frac{N \times R}{L \times T}$ in a highway scenario, where $N$ is the total number of active vehicles, $\mathrm{R}$ is the communication range, $\mathrm{L}$ is 
the length of the highway, $\mathrm{T}$ is the number of slots reserved for each area.

Figure 4 shows the merging collision rates of CF-MAC and DTMAC. As shown in this Figure, CF-MAC achieves less collision than DTMAC starting from AO > 0.7 where CFMAC works better as it eliminated the chance for the collision to some degree.

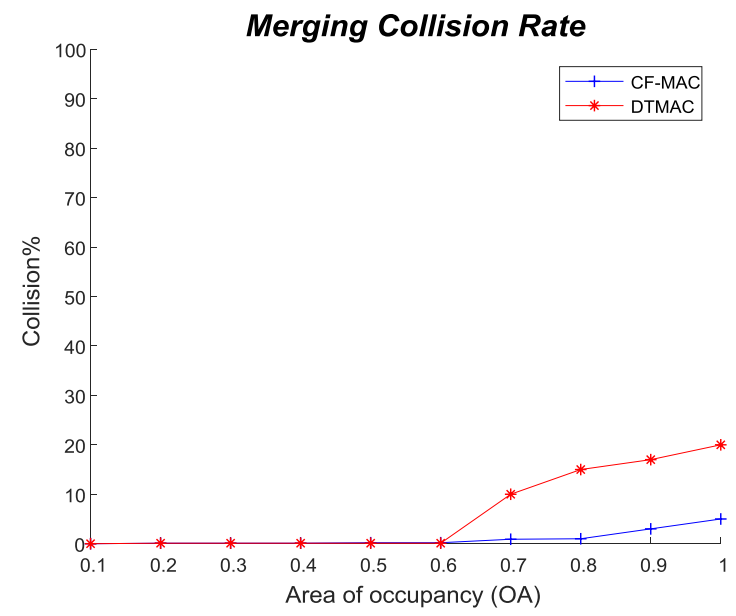

Fig 4: The rate of merging collision.

Figure 5 shows the access collision rates of CF-MAC and DTMAC. As shown in this Figure, CF-MAC achieves a smaller rate of access collisions than DTMAC starting, starting from $\mathrm{AO}(\geq 0.7)$. For instance, at $\mathrm{AO}=0.90$, the $\mathrm{CF}$ MAC protocol achieves an access collision rate of $4 \%$, in contrast to DTMAC which shows a rate of $20 \%$ (which means $16 \%$ higher collision than CF-MAC).

These results can be explained by the fact that DTMAC has achieved a higher rate of merging collision compared to CFMAC.

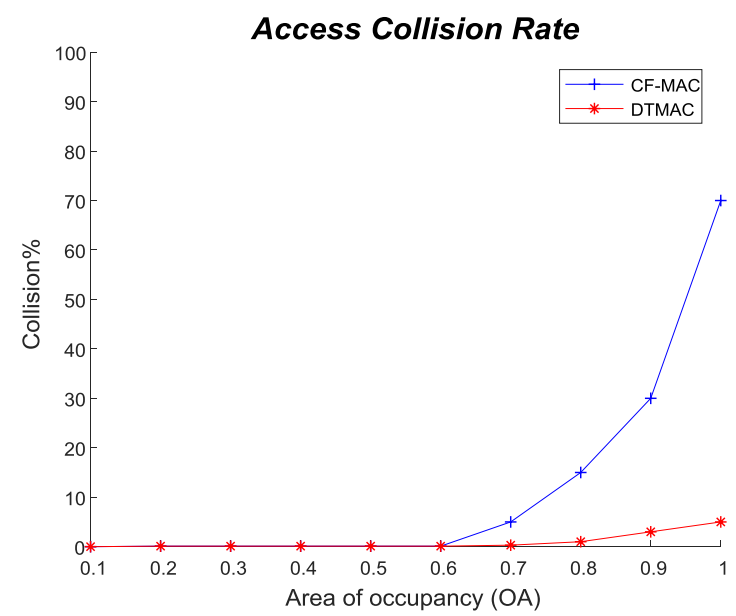

Fig 5: The rate of access collision.

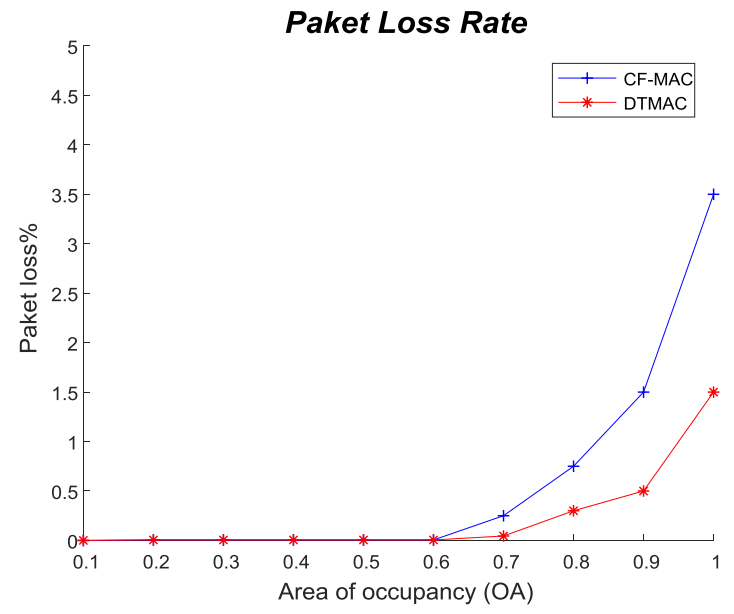

Fig 6: The rate of access collision.

The packet loss rates of the two are shown in Figure 6. For $\mathrm{AO} \leq 0.7$, the CF-MAC and DTMAC protocols have almost the same packet loss rate, while for AO > 0.7, CF-MAC starts to perform better than DTMAC. It can be seen that proposed protocol has the lowest packet loss rate, especially for a high $\mathrm{AO}$, due to its capability to deal with the merging collision problem. For instance, at $\mathrm{AO}=0.90$, the DTMAC protocol shows approximately $33.33 \%$ higher rate of packet loss than the CF-MAC protocol.

\section{CONCLUSION}

In this research a new collision free protocol to manage the channel access in Medium Access Control Sublayer (MAC) for the transmission is proposed, this protocol ensured a collision free management that enhanced the channel performance and increased the channel reliability.

The simulation results compared the proposed protocol CFMAC with DTMAC and showed that the CF-MAC scores better performance in terms of collision and packet loss ratio.

\section{ACKNOWLEDGMENT}

This research is funded by the Deanship of Research and Graduate Studies in Zarqa University /Jordan.

\section{REFERENCES}

[1] L. Kai, G. Jinhua, and L. Ning, "RAMC: A RSU-assisted multi-channel coordination MAC protocol for VANET," IEICE Trans., 2011.

[2] T. Luo, M. Motani, and V. Srinivasan, "CAM-MAC: A cooperative asynchronous multi-channel MAC protocol for ad hoc networks,", Networks Syst. 2006.

[3] R. Yadumurthy and M. Sadashivaiah, "Reliable MAC broadcast protocol in directional and omni-directional transmissions for vehicular ad hoc networks," Proc. 2nd, 2005.

[4] L. Zhou, B. Zheng, B. Geller, A. Wei, S. Xu, and Y. Li, "Cross-layer rate control, medium access control and routing design in cooperative VANET," Comput. Commun., 2008.

[5] H. Menouar, F. Filali, and M. Lenardi, "A survey and qualitative analysis of MAC protocols for vehicular ad hoc networks," IEEE Wireless. Commun., 2006.

[6] R. Baldessari, B. Bödekker, M. Deegener, and A. Festag, "Car-2-car communication consortium-manifesto," 2007. 
[7] I. C. S. L. M. Standards, "Wireless LAN medium access control (MAC) and physical layer (PHY) specifications," IEEE Stand. 802.11-, 1997.

[8] ETSI Standard, "Intelligent Transport Systems (ITS); European profile standard for the physical and medium access control layer of Intelligent Transport Systems operating in the $5 \mathrm{GHz}$ frequency band," 2009.

[9] G. Samara and W. Al-Salihy, "Security issues and challenges of vehicular ad hoc networks (VANET)," New Trends Inf., 2010.

[10] P. Engelstad and O. Østerbø, "Non-saturation and saturation analysis of IEEE 802.11 e EDCA with starvation prediction," Proc. 8th ACM Int., 2005.

[11] G. Samara and T. Alhmiedat, "Intelligent Emergency Message Broadcasting in VANET Using PSO," World Comput. Sci. Inf. Technol. J., vol. 4, no. 7, pp. 2221741, 2014.

[12] M. Van Eenennaam and A. Remke, "An analytical model for beaconing in VANETs," Conf. (VNC), 2012 ..., 2012 .

[13] S. Yuezhongyi, "Reliable and Secure Routing ADHOC Algorithm Based on Collaborative Monitor Nodes in VANETS," Int. J. Secur. Its Appl., 2015.
[14] M. Rani, "TDMA AND FDMA Integrated Hybrid Model for Communication Optimmization in VANET," IJRECE, 2015.

[15] Akram A. Almohammedi, Nor K. Noordin, Sabri Saeed, "Evaluating the Impact of Transmission Range on the Performance of VANET", International Journal of Electrical \& Computer Engineering, Vol. 6 Issue 2, p800-809, 2016.

[16] Samara, G.; Alsalihy, W. A. H. A.; Ramadass, S., "Increase Emergency Message Reception in Vanet", Journal of Applied Sciences, vol. 11, issue 14, pp. 26062612.

[17] G Samara, M Rasmi, "Deploying an Efficient Safety System for VANET", World of Computer Science and Information Technology Journal (WCSIT), Vol. 5, No. 3, 41-50, 2015.

[18] M. Hadded, A. Laouiti, and P. Muhlethaler, "An infrastructure-free slot assignment algorithm for reliable broadcast of periodic messages in vehicular ad hoc networks," VTC Fall, 2016.

[19] H. A. Omar, Weihua Zhuang, and Li Li, "VeMAC: A novel multichannel MAC protocol for vehicular ad hoc networks," in 2011 IEEE Conference on Computer Communications Workshops (INFOCOM WKSHPS), 2011, pp. 413-418. 